\title{
Erstes Positionspaper der DeGIR zur Prostataarterienembolisation
}

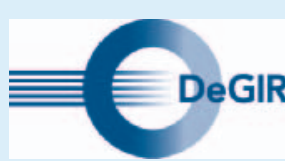

DEUTSCHE GESELLSCHAFT FÜR INTERVENTIONELLE RADIOLOGIE UND MINIMAL-INVASIVE THERAPIE

in der Deutschen Röntgengesellschaft e.V.

Das erste Positionspapier (White Paper) der Deutschen Gesellschaft für Interventionelle Radiologie und minimal-invasive Therapie (DeGIR) ist am 2 . Juli 2020 online erschienen. In der von der DeGIR-Lenkungsgruppe Wissenschaft und Forschung initiierten Arbeit nimmt die Gesellschaft zum Verfahren der Prostataarterienembolisation eindeutige Stellung.

Die Prostataarterienembolisation (PAE) steht seit einigen Jahren als Behandlung des benignen Prostatasyndroms (BPH) zur Verfügung und muss sich in diesem Kontext mit dem Goldstandard der transurethralen Resektion (TUR-P) vergleichen lassen. In der vorgelegten Arbeit beschreiben die Autoren die Indikationen, die Methodik und die
Ergebnisse der PAE. Insbesondere die Ergebnisse werden im wissenschaftlichen Kontext mit der TUR-P ausführlich bewertet. Auf Basis der international vorliegenden Daten wird die PAE in Großbritannien vom NICE (National Institute for Health and Care Excellence) als eine alternative zur TUR-P empfohlen, da in Bezug auf die subjektive Beschwerdesymptomatik die PAE im Vergleich zur TUR-P vergleichbare Ergebnisse liefert. Allerdings ist die PAE in Hinsicht auf die subvesikale Desobstruktion der TUR-P unterlegen.

In der Bundesrepublik Deutschland bieten immer mehr radiologische Abteilungen diesen Eingriff an, wobei eine interdisziplinäre Zusammenarbeit mit den urologischen Kolleginnen und Kollegen empfehlenswert ist.
Trotz der immer besser werdenden Datenlage ist bei manchen Kolleginnen und Kollegen die Skepsis allerdings nicht unerheblich. Dies schlägt sich auch in den aktuell überarbeiteten Guidelines der ESU (European Society of Urology) und der AUA (American Urological Association) nieder. Auch die noch schwierige Vergütungssituation wird im Positionspaper der DeGIR ausführlich beschrieben.

Das Positionspapier ist online frei verfügbar:

https://www.thieme-connect.com/ products/ejournals/abstract/10.1055/ a-1183-5438

Der Beitrag wurde von Prof. Dr. Christian Habermann, DeGIR-Team Öffentlichkeitsarbeit, verfasst. 